\title{
Anabases
}

ANABASES Traditions et réceptions de l'Antiquité

\section{Pensée politique, surinterprétation et histoire classique. La réception de Tacite à l'âge moderne}

\section{Saúl Martínez Bermejo}

\section{(2) OpenEdition}

1 Journals

Édition électronique

URL : http://journals.openedition.org/anabases/1901

DOI : 10.4000/anabases. 1901

ISSN : 2256-9421

\section{Éditeur}

E.R.A.S.M.E.

\section{Édition imprimée}

Date de publication : 1 mars 2011

Pagination : 276-281

ISSN : 1774-4296

\section{Référence électronique}

Saúl Martínez Bermejo, «Pensée politique, surinterprétation et histoire classique. La réception de

Tacite à l'âge moderne », Anabases [En ligne], 13 | 2011, mis en ligne le 01 mars 2014, consulté le 20 octobre 2019. URL : http://journals.openedition.org/anabases/1901 ; DOI : 10.4000/anabases.1901

Ce document a été généré automatiquement le 20 octobre 2019.

(c) Anabases 


\title{
Pensée politique, surinterprétation et histoire classique. La réception de Tacite à l'âge moderne
}

\author{
Saúl Martínez Bermejo
}

1 Tacite était considéré comme un «maître de la politique » à l'âge moderne. Pour Filippo Beroaldo, chargé en 1515 de la première édition des œuvres «complètes» de l'historien, Tacite surpassait Salluste et Tite-Live, sa narration étant spécialement utile aux nobles, princes et empereurs ${ }^{23}$. Son style extrêmement concis et la manière dont Tacite aborde les enjeux du pouvoir, les dispositions des empereurs, les dénonciations et dissimulations, les causes des événements, etc. surprenaient les lecteurs de la Renaissance, qui les confrontaient avec les récits, moins perspicaces et pénétrants, d'autres historiens. En 1517, Andrea Alciato soulignait aussi les enseignements «stoïciens » que contenait, de son point de vue, l'œuvre de Tacite : des exemples de constance, de courage et de la manière de bien agir sous les mauvais princes ${ }^{24}$. Cette chaîne ininterrompue d'éloges - et d'interprétations - se poursuit tout au long du XVI ${ }^{e}$ siècle et se consolide fermement dans les éditions de l'érudit flamand Juste Lipse. Dès sa première version, en 1574, Juste Lipse amplifie les topiques précédents, soulignant aussi dans sa dédicace à Maximilien II les connexions entre le récit des débuts de l'Empire romain et la situation politique contemporaine. Lipse met l'accent sur l'utilité de Tacite pour fournir des exemples aux lecteurs de l'âge moderne, en vertu de la similitudo temporum ${ }^{25}$.

2 Les réflexions de ces trois éditeurs ne représentent cependant qu'une petite partie des innombrables éloges de Tacite, et du passage de l'histoire classique à la pensée politique : une translatio qui s'opère par le biais des exemples et de la comparaison, et qui constitue une des caractéristiques plus intéressantes et singulières de la réception de Tacite aux XvI et $\mathrm{XVII}^{\mathrm{e}}$ siècles. Ce terme - «réception »-, veut ici renvoyer à un ensemble de théories littéraires classées sous le nom de Rezeptionsästhetik. Un certain nombre de spécialistes, comme Hans Robert Jauss, ont souligné l'importance du rôle du lecteur dans la construction du sens du texte, le désignant comme un troisième pôle 
avec la même « importance » que ceux que représentent le texte et l'auteur ${ }^{26}$. En effet, l'histoire de la réception de Tacite à l'âge moderne peut être conçue comme une histoire des changements successifs de l'«horizon d'attente ». Les «schémas " tracés par les éditeurs dans leurs présentations liminaires constituent un exemple de cet horizon qui était défini, entre autres, par la théorie rhétorique du moment, les conceptions et traités sur l'histoire et ses applications, et les pratiques de lecture et d'annotation apprises à l'école et dans les universités. Dans une telle perspective, l'objectif de la recherche historique est de récupérer les interprétations passées de Tacite, mais aussi des modalités de lecture disparues et aujourd'hui incompréhensibles, ainsi que leurs conséquences imprévues.

3 En explorant les caractéristiques particulières de l'usage de Tacite, on découvre que son utilisation politique s'inscrit dans une tendance à la surinterprétation de ses textes. Umberto Eco a défini cette notion par le truchement de l'exemple de la relation entre les mots « pendant » et « crocodile ». Certes, argumente Eco, les deux apparaissent dans la phrase qu'il venait d'utiliser comme exemple; mais déduire «le maximum possible de cette relation minime », et voir "dans mon exemple un secret caché auquel je me réfère ", constitue tout simplement une interprétation paranoïaque, une surinterprétation ${ }^{27}$. Les nombreuses références à l'obscurité (tant sur le plan stylistique que sur celui des contenus) des textes de Tacite, à la nécessité de disposer d'interprètes judicieux, au caractère occulte de certains passages (un trésor de sentences à découvrir, des fleurs à conserver) constituent mélange de clichés, de notions rhétoriques et d'appels à une surinterprétation du texte. Les divers commentateurs, traducteurs et éditeurs demandaient à leurs lecteurs d'observer les textes de Tacite avec le plus grand soin et avec la plus grande précision possibles, et les incitaient à les analyser attentivement, donc à les surinterpréter.

4 Fondé sur des techniques qui favorisent la mise en avant d'extraits du texte (des "sentences », ces «gemmes » dont Juste Lipse parle en préface de ses éditions, ou les citations les plus variées), ce "mode de lecture » détachait les extraits de leur contexte et les préparait pour une application aux réalités contemporaines. Pour le traducteur et commentateur espagnol Baltasar Álamos de Barrientos, les leçons les plus importantes étaient réservées à ceux «qui le lisent et le ruminent avec l'intention d'extraire la substance qu'il contient et qu'il cache ${ }^{28} »$. Nicolas Abraham Amelot de la Houssaye incarne un type spécifique de commentateurs de Tacite, ceux qui «comme politiques, sans s'arrêter à sa frase, ni à la diction se sont étudiés à pénétrer les mistères, \& les secrets de l'Art-de-gouverner, dont il est le Maître, \& l'Oracle universel, depuis de quinze-cens $a^{29}{ }^{29}$ ». Les livres de commentaires, enfin, (d'Annibale Scoto ou Scipione Ammirato à Christophe Forstner) nous offrent des preuves de la matérialisation de ce mode de lecture: les extraits des œuvres originales sont suivis d'un commentaire détaillé, digressif au minimum, enclin à la surinterprétation en maintes occasions ${ }^{30}$.

De Arcanis rerum publicarum libri sex (1605) est un des plus célèbres livres de commentaires de Tacite. Dans ce livre, Arnold Clapmarius consacrait un chapitre entier au commentaire du passage Histoires I, $4:$ : Car un secret d'État venait d'être divulgué : on pouvait faire un empereur ailleurs qu'à Rome ${ }^{31}$. » Ce mode de lecture, qui implique un évident détachement par rapport à la logique du récit - maintenant réduit à de petites portions, à des leçons prêtes à être employées -, était aussi celui de l'érudit et traducteur anglais de Tacite, Henri Saville ${ }^{32}$. Clapmarius considère la phrase tacitéenne comme une règle, une norme universelle (ou " universellisable») du politique ; Savile, 
plus prudent, discute toutes les apparitions du terme arcana imperii (ou similaires) dans les ouvrages de Tacite et indique ses multiples acceptions, mais il cède finalement à l'interprétation de cette sentence comme si Tacite avait eu l'intention de donner au monde un principe directeur des événements politiques. Il existait cependant, à l'époque, des opinions contraires à cette manière de lire: Anthony Grafton a indiqué que Johannes Kepler " insistait à traiter l'œuvre de Tacite comme un tout organique » et encourageait ses lecteurs à ne pas y trouver simplement des enseignements élémentaires, prêts à être utilisés. Au moment d'interpréter le passage Histoires I, 4, Kepler en vient à considérer que Tacite décrit simplement une situation historique concrète, et non pas une règle occulte de gouvernement ${ }^{33}$. Mais les lectures fragmentées et les modes de surinterprétation étaient majoritaires.

6 Face à cette modalité de lecture, on peut être amené à dénoncer l'interprétation abusive de Tacite, comme l'a fait, par exemple, le philologue espagnol Francisco Sanmartí Boncompte, qui considère que ceux qui ont contemplé Tacite comme un «oracle politique» l'ont fait d'un point de vue «absolument flou ${ }^{34} »$. On peut aussi essayer de trouver, ou de reconstruire, une interprétation «correcte ${ }^{35}$ ». En même temps, en historien, on peut montrer comment cette modalité d'interprétation constitue une clé pour repenser l'évolution de la politique, discipline de conceptualisation et de définition du monde social ${ }^{36}$. En outre, la surinterprétation peut parfois affecter la notion même de "texte classique » : à l'âge moderne, il ne s'agissait pas de textes inamovibles d'une époque déjà oubliée, mais d'un outillage mental à disposition de tous, au quotidien. La relation entre deux époques séparées par plus de quinze siècles était conçue de manière fluide, ce qui permettait l'application de l'Antiquité au présent. Ma thèse, intitulée Tacite lu. Pratiques de lecture et fondements intellectuels de la réception de Tacite à l'âge moderne ${ }^{37}$, étudie la réception de cet auteur comme moyen d'accéder à la compréhension du développement de la politique à l'âge moderne.

7 La première des deux parties a pour but de proposer une vision systématique du processus de création du sens dans les textes de Tacite. En utilisant une approche inspirée par l'histoire de la lecture, je rends compte du rôle joué par divers participants dans l'interprétation des textes (celle du lecteur qui annote sa copie des œuvres de Tacite, celles de l'éditeur, de l'imprimeur ou du traducteur) et les formes matérielles des livres imprimés. Cette analyse constitue la base du questionnement sur l'utilisation politique de Tacite à l'âge moderne. Dans la seconde partie, la structure thématique cède le pas à une approche chronologique et par pays, et à une méthodologie plus proche de l'histoire de la pensée politique. Cette partie ne recense pas seulement l'emploi des œuvres de Tacite dans divers contextes politiques européens, mais cherche aussi à expliquer la nature de la pensée politique à l'âge moderne, ses caractéristiques comme discipline, et ses fondements intellectuels.

8 Même s'il s'agit d'une activité qui «se déroule sous nos nez ", selon l'expression de Robert Darnton, la lecture reste un phénomène étrange et mystérieux. Les témoignages historiques de lecture sont rares et difficiles à récupérer, mais ils offrent une information précieuse. Dans le premier chapitre, j'analyse les types de marques que les lecteurs modernes ont laissés sur plusieurs exemplaires de Tacite, en effectuant dans la foulée une description dense de deux séries d'annotations marginales. Les premières font apparaître une lecture universitaire "standard", produite à Salamanque vers 1604. L'autre série de notes, conservées dans ce cas sur les marges d'un exemplaire 
d'une traduction castillane, constitue un témoignage exceptionnel de lecture "politique». Le deuxième chapitre développe le sujet précédent et étudie des annotations plus complexes sur Tacite, notamment celles des livres de lieux communs, dans lesquels j'ai choisi à nouveau deux cas concrets pour mieux illustrer les différentes modalités de lecture. Ce panorama des divers "témoignages» de lecture est accompagné de constantes références à la théorie de la lecture et de l'annotation à l'âge moderne. Les préceptes de la rhétorique moderne permettent de comprendre la logique interne des annotations de lecture et l'utilisation ultérieure des morceaux de texte recueillis pour composer une nouvelle argumentation, pour servir de guide à l'action, etc.

9 Le troisième chapitre est consacré aux "médiations" dans la réception du texte. J'entends par «médiations » ou " conditions » de réception toutes les caractéristiques matérielles des livres, du format à la table des matières, en passant par les approbations et privilèges, les présentations du texte dans les préliminaires, frontispices, illustrations, résumés de chapitres, annotations marginales imprimées, chronologies, notes en bas de page, etc. Les diverses modifications du sens que provoque la somme de ces dispositifs constituent une clef de lecture importante pour comprendre l'interprétation de Tacite, et les phénomènes de circulation intellectuelle au niveau européen. Dans ce chapitre, j'aborde aussi la question de la popularisation du texte classique latin. J'analyse de façon détaillée la perception cultivée de la portée de Tacite, liée aux craintes et préjugés concernant la vision populaire de la politique et du gouvernement.

10 La traduction constitue une des médiations les plus significatives dans le processus de réception. C'est un moyen exceptionnel de dissémination et de transformation du texte original, et fournit l'occasion de l'insérer dans des contextes imprévus. Le quatrième chapitre est consacré exclusivement à comprendre les changements subis par les textes au cours de la traduction, et les implications historiques et politiques de ces modifications. J'y relève les rapprochements et distanciations entre les événements de l'histoire romaine racontés par Tacite et la réalité de l'âge moderne; les matières qui favorisent la comparaison et l'application au présent et celles qui provoquent une critique, parfois brutale, de l'Antiquité et ses coutumes. Une version revue et augmentée de ce chapitre est devenue un petit livre, intitulé Translating Tacitus. The reception of Tacitus in the vernacular languages of Europe, 16th-17th centuries (Éd. PlusUniversità de Pisa, 2010).

11 Le chapitre 5 analyse le «mode d'emploi » de Tacite dans la pensée politique espagnole sous Philipe II et Philipe III. J'y examine les divers auteurs qui recommandent ou critiquent Tacite, et la nature de cette confrontation. En particulier, je mets en avant la flexibilité et la capacité d'innovation des penseurs espagnols face aux difficultés et défis de la modernité. La discussion se centre aussi sur les implications «scientifiques » de la méthode d'application des histoires de Tacite à la réalité des $\mathrm{XVI}^{\mathrm{e}}$ et $\mathrm{XVII}$ siècles. Les chapitres 6 et 7 sont consacrés à la France et à l'Angleterre respectivement. Dans mon exposé, je souligne à nouveau l'importance de l'histoire et de la théorie de l'histoire comme cadre de référence de la pensée politique. Pour la France, j'analyse le Methodus ad facilem historiarum cognitionem de Jean Bodin, un des plus importants «arts historiques » de la Renaissance. Grâce à cette source, on comprend mieux les méthodes de lecture des textes historiques, ainsi que la manière d'extraire des passages et de les classer. Un panorama général des éditions et traductions françaises sert à montrer 
l'emploi «absolutiste» et les diverses utilisations (didactiques, stylistiques, etc.) de Tacite. Ce chapitre constitue la base de mon projet de recherche actuel, qui vise à approfondir mes investigations sur la réception de Tacite en France.

Pour l'Angleterre, j'ai analysé les divers essais et traités sur l'utilisation de l'histoire, et les mentions de Tacite dans ces textes. Je centre une grande partie de mon analyse sur l'emploi de Tacite par le comte d'Essex pour défendre sa vision de l'armée et de la politique, aussi bien que pour se donner une image publique d'homme sage et expert en matière de gouvernement. Cette approche se combine avec une étude minutieuse de la traduction de Henry Savile, l'érudit chargé d'accommoder Tacite aux intérêts d'Essex comme lecteur de Tacite.

Le huitième et dernier chapitre laisse de côté les implications idéologiques ou doctrinales du tacitisme pour analyser les fondements des argumentations construites au moyen de ces textes et la façon dont les méthodes de lecture de l'histoire affectent la conception de la politique comme discipline. J'explore cette question à travers la place accordée aux livres concernant Tacite dans les bibliothèques modernes et dans les portraits de certains « intellectuels ». Mes réflexions finales sont consacrées à l'analyse du rapport de la politique avec d'autres disciplines comme la théologie ou la littérature et aux implications rationalistes de ces méthodes qui définissent la politique comme une activité à la portée de l'intellect humain, avec des normes de fonctionnement relativement accessibles et modélisables.

\section{NOTES}

23. Tacite, P. Cornelii Taciti Libri quinque noviter inventi atque cum reliquis ejus operibus editi, Rome, Stephanum Guillereti de Lothoringia, 1515, dédicace à Léon X.

24. Tacite, Libri quinque noviter inventi, Milan, Alessandro Minuziano, 1517, dédicace à Galeazzo Visconti.

25. Tacite, Historiarum et Annalium libri qui exstant, Anvers, Christophoro Plantino, 1574, dédicace à Maximilien II.

26. H.R. JAUSS, "La historia de la literatura como provocación de la ciencia literaria", en La historia de la literatura como provocación, Barcelone, Péninsule, 2000, p. 137-193 (éd. or. Literaturgeschichte als Provokation der Literaturwissenschaft, Constance, 1967). Voir aussi W. ISER, El acto de leer. Teoría del efecto estético, Madrid, Taurus, 1987 ; J.A. MAYORAL (éd.), Estética de la recepción, Madrid, Arco Libros, 1987 et R. WARNING (éd.), Estética de la recepción, Madrid, Visor, 1989.

27. U. ECO, S. COLLINI (éd.), Interpretation and overinterpretation, Cambridge, Cambridge University Press, 1992, p. 48.

28. B. ÁLAMOS DE bARRIENTOS, Tácito español ilustrado con aforismos, Madrid, Luis Sánchez, 1614, dédicace au duc de Lerma.

29. N. A. AMELOT DE LA HOUSSAYE, Tibère. Discours politiques sur Tacite, Amsterdam, héritiers de Daniel Elzevir, 1683, préface. 
30. A. sсото, In P. Cornelii Taciti Annales, et Historias comentarii ad politicam, \& aulicam rationem praecipue spectantes, Rome, Bartolomeo Grassi, 1589 ; S. AMMIRATO, Discorsi del signor Scipione Ammirato sopra Cornelio Tacito, Florence, Filippo Giunti, 1590 ; C. FORSTNER, Christophori Forstneri In XVI libros Annalium, (quatenus extant) C. [aji] Cornelii Taciti notae, Francfort, Johannes Beyerus, 1662. La liste des commentateurs est à établir. Pour les commentaires latins, voir R. W. ULERY, “Tacitus”, in F.E. CRANZ, V. BROWN, P.O. KRISTELLER (éd.), Catalogus translationum et commentariorum. Mediaeval and Renaissance Latin translations and commentaries, annotated lists and guides, Washington, Catholic University of America Press, 1989, vol. 6, s.v.

31. A. CLAPMARIUS, De Arcanis rerum publicarum libri sex, Lugduni Batavorum, Iacobum Marci, 1644, http://www.uni-mannheim.de/mateo/camenahist/autoren/ clapmar_hist.html (site consulté le $1^{\mathrm{er}}$ novembre 2010), Tacite, Histoires I, 4, traduction par H. GOELZER, Les Belles Lettres, Paris, 1921.

32. Tacite, The end of Nero and beginning of Galba. Four books of the Histories of Cornelius Tacitus. The life of Agricola, Oxford, Ioseph Barnes [et Londres, R. Robinson] for Richard Wright, 1591 (Traduction et édition de H. SAVILE), Annotations upon the first book of the Histories, p. 6. A. GRAFTON, Commerce with the classics : ancient books and renaissance readers, Ann Arbor, University of Michigan Press, 1997, p. 206, considère cette manière comme typique des « interprètes habituels » de Tacite.

33. A. GRAFTON, Commerce with the classics, p. 207-208.

34. F. SANMARTí BONCOMPTE, Tácito en España, Barcelone, Consejo Superior de Investigaciones Científicas, 1951, p. 116.

35. Exemples de ce type de critique philologique chez R. SYME, Tacitus, 2 vols, Oxford, Oxford University Press, 1958 et A.J. woodman, Tacitus reviewed, Oxford, Oxford University Press, 1998.

36. J.G.A. Pососк, Politics, language \& time. Essays on political thought and history, Chicago, Chicago University Press, 1989.

37. S. MARTínez BERMEJO, Tácito leído. Prácticas lectoras y fundamentos intelectuales de la recepción de Tácito en la edad moderna, Madrid, Universidad Autónoma de Madrid, http:// hdl.handle.net/10486/4336.com (site consulté le $1^{\mathrm{er}}$ novembre 2010).

\section{AUTEUR}

\section{SAÚL MARTÍNEZ BERMEJO}

Département d'Histoire Moderne, Université Autonome de Madrid saul00@gmail.com 